Maternal polyhydramnios has been noted in several cases, but the explanation of its occurrence remains obscure. ${ }^{3}$ As a rule the fact that the tumour is of renal origin can be established by intravenous urography, which shows displacement and distortion of the collecting system. However, an abdominal neuroblastoma, which is a much commoner congenital neoplasm, can invade the kidney and produce a very similar pyelogram. The distinction can be made by estimation of the urinary excretion of catecholamine, which is raised with a neuroblastoma but not with a kidney tumour. J. Waisman and P. H. Cooper ${ }^{4}$ emphasized the vascular sinusoids of the mesenchymal kidney tumour of the newborn and their angiographic appearance in injected specimens, but W. E. Berdon and colleagues ${ }^{5}$ did not find that transumbilical aortography was helpful in distinguishing a mesenchymal tumour from a nephroblastoma, since both showed similar production of new blood vessels.

The mesenchymal renal tumour has been variously described as fibrosarcoma, ${ }^{6}$ leiomyoma, ${ }^{7}$ and fetal renal hamartoma. ${ }^{5}$ R. P. Bolande and colleagues, ${ }^{8}$ who were first to emphasize that the tumour is distinct from, though possibly related to, the nephroblastoma, coined the term mesoblastic nephroma. The most important practical point is that, even though these neoplasms are unencapsulated and even though microscopy may show immaturity of nuclear structure and much mitotic activity, their behaviour, with the single exception of an atypical case reported by D. Walker and G. A. Richard ${ }^{9}$ has been that of benign tumours, and neither local recurrence nor distant metastases have been reported after surgical excision. In Walker and Richard's patient the tumour was noted at birth by the mother and explored at the age of 3 months. Though histology showed the usual leiomyomatous appearance, the tumour had infiltrated the perinephric fat and there was much necrosis and cyst formation. The child had a fatal recurrence of the neoplasm four months after nephrectomy.

It is now accepted that treatment of a typical mesenchymal renal tumour should be limited to nephrectomy without the use of postoperative irradiation or cytotoxic chemotherapy, which are employed in the management of nephroblastoma in the older child but which carry higher complication rates when given to infants. ${ }^{1011}$ It is noteworthy that a true nephroblastoma is generally of low-grade malignancy in children under 1 year of age and, unless there is clearly extension of the tumour beyond the kidney, treatment in this age group should likewise be surgical only.

Just where the mesenchymal neonatal tumour fits into the classification of kidney neoplasms is uncertain. Some authors have considered it to be a separate and distinct entity, ${ }^{5}$ while others have regarded it as a differentiated form of nephroblastoma. ${ }^{8} \mathrm{~J}$. B. Beckwith, ${ }^{2}$ who favours the latter view, suggests that there may be some biochemical peculiarity of the fetus which influences the differentiation of an incipient kidney tumour towards a fibromatous or myomatous pattern.

Why are these benign mesenchymal tumours encountered only in very young babies and not later ? It would seem unlikely that every case would be diagnosed and treated promptly and that none would escape early detection only to be found subsequently in the older infant or child. This unanswered question suggests that a mesenchymal neoplasm may progress with time to form a typical nephroblastoma, and it is possible that Walker and Richard's ${ }^{9}$ case constitutes the missing link between the two types of tumour. Certainly their report shows that, while a renal tumour in a newborn baby is likely to be benign, delay over its removal can be dangerous.

1 Favara, B. E., Johnson, W., and Ito, J., Cancer, 1968, 22, 845.

2 Beckwith, J. B., fournal of Pediatric Surgery, 1970, 5, 405.
${ }^{3}$ Richmond, H., and Dougall, A. J., Fournal of Pediatric Surgery, 1970, 5, 413.

4 Waisman, J., and Cooper, P. H., Fournal of Pediatric Surgery, 1970, 5, 407. Berdon, W. E., Wigger, H. J., and Baker, D. H., American fournal of

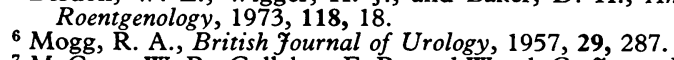

McCune, W. R., Galleher, E. P., and Wood, C., fournal of Urology, 1964, $91,646$.

${ }^{8}$ Bolande, R. P., Brough, A. J., and Izant, R. J., Pediatrics, 1967, 40, 272.

9 Walker, D., and Richard, G. A., Fournal of Urology, 1973, 110, 352.

10 Lascari, A. D., Fournal of Pediatrics, 1972, 80, 337.

11 Berdon, W. E., Baker, D. H., and Boyer, J., American fournal of Roentgenology, 1965, 93, 545.

\section{Vision on the Road}

Do we need more rigorous visual screening for drivers of private motor vehicles? The Association of Optical Practitioners arranged a symposium to discuss this question on 17 October at the Royal College of Surgeons, and coverage by press and television was organized for it. The issue is of importance, and the A.O.P. is to be congratulated on its airing, even if the panel of speakers was lop-sided in that, though the majority of them were naturally enough sighttesting opticians, no ophthalmologist was included.

During the symposium speakers frequently stated that accidents are caused by bad vision and that there should be routine screening, but no real evidence was offered to substantiate these views. In fact the main research on this subject, carried out in the U.S.A. on over 17,000 drivers, showed that their frequency of accidents correlated with good vision rather than bad vision, which suggests that those with poor vision took more care. And a summary by G. T. W. Cashell ${ }^{1}$ concluded: "There is no evidence so far to lead one to assume that eye defects in drivers may be regarded as having an effect on road accidents. The driver with a defect of vision of which he is aware is usually a careful driver and compensates for it; the real culprits are those with normal vision who do not pay visual attention to driving and whose judgement and ability are impaired by many extrinsic factors." In those countries where extensive screening has been adopted there has been no apparent reduction in accident rates. Indeed, at this symposium Dr. Ivan Brown, of the Medical Research Council's Applied Psychology Unit at Cambridge, emphasized that it is "the brain behind the eyes rather than the eyes themselves" which really matter. Hence the frequency of accidents in fog by drivers who keep too close together owing to their judgements of distance being impaired.

Several interesting facts emerged at the meeting. An excellent report by $\mathrm{Mr}$. H. Philips showed that special nightdriving glasses, polarizing lenses, and windscreens that were heat-absorbent or had sprayed-on tints were never beneficial and usually harmful, especially by delaying the recovery time after overexposure to glare. Other speakers suggested that as well as regular checks on central vision drivers should have their visual fields and even night vision assessed. But a threeyearly perimetry on all drivers would hardly disclose those sudden major impairments of field which alone might be dangerous (for people with long-standing field-losses will have learnt to compensate by frequent eye movements), and the testing of night blindness, even if this were found to be a factor in car accidents, is a tedious and complicated undertaking which would be impracticable on a national scale.

The Road Research Laboratory had indeed initiated extensive trials and its findings are awaited. But in the absence of any evidence that impaired vision is a significant cause of road accidents the view of the Faculty of Ophthalmologists is sound, 
namely, that the present visual restrictions are adequate. There is no occasion to waste our limited resources by subjecting our driving population to regular eye examinations or repetitive visual checks.

${ }^{1}$ Cashell, G. T. W., Transactions of the Ophthalmological Societies of the United Kingdom, 1966, 86, 617.

\section{More about Chenodeoxycholic Acid}

After the initial announcement by R. G. Danzinger and his colleagues $^{1}$ that cholesterol gallstones could be dissolved by oral chenodeoxycholic acid therapy further studies have confirmed the effectiveness of this bile acid in the medical treatment of gallstones. But at the same time they have shown that the treatment can carry some risk.

In the preliminary report seven women with gallstones received from 0.75 to $4.5 \mathrm{~g}$ chenodeoxycholic acid daily. The gallstones disappeared in one patient, became smaller in three others, and were unchanged in the remaining three. Encouraged by these results J. L. Thistle and A. F. Hofmann ${ }^{2}$ embarked on a single-blind, controlled therapeutic trial of chenodeoxycholic acid, cholic acid, and placebo in 53 patients with asymptomatic stones. The dose of chenodeoxycholic acid averaged $18 \mathrm{mg} / \mathrm{kg}$ of body weight per day. Cholecystograms were obtained at the beginning of therapy and again after six months, at which time 11 of the 18 patients who had received chenodeoxycholic acid showed a reduction in the size or the number of gallstones. No change was observed in those patients taking cholic acid or placebo. Of 13 patients with radio-opaque stones only two showed a partial response to the chenodeoxycholic acid.

Other workers studying this bile acid have had comparable results. Thus G. D. Bell and colleagues, ${ }^{3}$ giving a daily dose of 0.75 to $1.0 \mathrm{~g}$, reported that after six months the stones had disappeared in three of 12 patients who had functioning gall bladders, while in three others there was appreciable reduction in stone size. $\mathrm{O}$. James and colleagues ${ }^{4}$ gave a similar dose and observed the dissolution of gallstones or considerable diminution in size in four of 11 patients. An improvement in the patients' symptoms may have been associated with dissolution of the gallstones. ${ }^{4}$ On the other hand there was no return of function in gallbladders which were radiologically nonfunctioning at the beginning of treatment. ${ }^{3}$

There is much concern over the potential toxicity of chenodeoxycholic acid and its metabolites. Chenodeoxycholic acid is a primary dihydroxy bile acid which undergoes 7- $\alpha$ dehydroxylation in the colon with the formation of lithocholic acid. Lithocholic acid is known to be a potent hepatotoxic agent when fed to a variety of laboratory animals, though some are apparently immune to its effects. Thus feeding large doses of chenodeoxycholic acid to rhesus monkeys induces a severe liver lesion comprising proliferation of bile ducts and periportal infiltration. Damage to the fetal liver has been reported. ${ }^{6}$ Perhaps therefore it is not surprising that all three groups using this substance have encountered altered liver function tests in patients under study. The most frequent abnormality was a rise in the concentration of serum aspartate transaminase. It usually occurred during the initial stages of therapy, and the concentration returned to normal during the course of treatment. It is of some interest that no such changes were observed in patients receiving cholic acid. ${ }^{2}$ The serum alkaline phosphatase activity also rose. James and colleagues ${ }^{4}$ have observed a rise in the concentration of total serum bile acid, but the important question regarding which bile acids were concerned remains to be answered. Thistle and Hofmann ${ }^{2}$ performed liver biopsies on 11 patients; eight were reported as normal, but fatty change was present in one and a periportal fibrous reaction was noted in two. In another study of the liver morphology of patients on chenodeoxycholic acid biopsies were obtained from 10 patients. ${ }^{7}$ The majority were normal but a few showed steatosis or non-specific changes. Thus far no lesion comparable to that reported in the monkey has been observed. Nonetheless the possibility of damage to the liver cannot be ignored, and the use of this drug in selected patients must be accompanied by rigorous clinical surveillance.

An interesting consequence of chenodeoxycholic acid therapy may be a reduction in serum lipids. ${ }^{8}$ Serum triglyceride values fell from average pretreatment levels of 118 to $95 \mathrm{mg} / 100 \mathrm{ml}$, whereas serum cholesterol concentrations were unchanged. The mechanism for this action is yet to be explained.

In general patients with cholesterol gallstones secrete a bile saturated or supersaturated with cholesterol. The underlying hepatic abnormality may be a dual defect of a reduced size of the bile acid pool and increased secretion of biliary cholesterol. ${ }^{9}$ The administration of bile acids would be expected to cause the bile to become undersaturated, thereby enhancing the solubility of cholesterol and encouraging the dissolution of gallstones. The initial study of Thistle and L. J. Schoenfield ${ }^{10}$ suggested that this was so and more recently both Thistle and Hofmann ${ }^{2}$ and Bell and colleagues ${ }^{3}$ observed an improvement in bile composition in patients on chenodeoxycholic acid. It is therefore surprising that in the study of James and colleagues $^{4}$ no improvement in the cholesterol-holding capacity of the bile was apparent. Feeding chenodeoxycholic acid would be expected to expand the total bile salt and chenodeoxycholic acid pools with corresponding reduction of the cholic and deoxycholic acid pool. However, T. C. Northfield and colleagues $^{11}$ have reported that the major effect of this bile acid is to decrease the rate of cholesterol secretion into bile in relation to the output of bile acids and phospholipids, and that this may be the major reason for the bile becoming undersaturated in cholesterol.

The chenodeoxycholic acid story is just beginning to unfold and at present more questions are being posed than answered: which patients are likely to respond, what is the optimal dose, what is the correct procedure once stones have been dissolved, is the liver damaged, what is the mode of action, and why does cholic acid not work? But the initial studies have shown convincingly that the dissolution of gallstones is at last becoming a feasible prospect.

1 Danzinger, R. G., Hofmann, A. F., Schoenfield, L. J., and Thistle, J. L., New England fournal of Medicine, 1972, 286, 1 .

2 Thistle, J. L., and Hofmann, A. F., New England fournal of Medicine, $1973,289,655$

3 Bell, G. D., Whitney, B., and Dowling, R. H., Lancet, 1972, 2, 1213

4 James, O., Cullen, J., and Bouchier, I. A. D., Gut, 1973, 14, 827. Bell, G. D., Whitney, B., Lewis, B., Thwe, M., and Dowling, R. H. Quarterly fournal of Medicine, 1973, 42, 824

- Heywood, R., Palmer, A. K., Foll, C. V., and Lee, M. R., Lancet, 1973, 2 , 1021 .

7 James, O. F. W., Scheuer, P. J., and Bouchier, I. A. D., Digestion, 1973, 8 432.

${ }^{8}$ Bell, G. D., Lewis, B., Petrie, A., Dowling, R. H., British Medical fournal, 1973, 3, 520. Grundy, S. M., Metzger, A.

10 Tnisestigation,

Thistle, J. L., and Schoenfield, L. J., New England Fournal of Medicine, 1971, 284, 177.

11 Northfield, T. C., LaRusso, N. F., Thistle, J. L., and Hofmann, A. F., Gastroenterology, 1973, 64, 780 . 